\title{
Lithium plus Olanzapine as One of the Most Effective Combinations for Bipolar Disorder. A Case Report and a Concise Review of the Literature
}

\author{
Miguélez Rodríguez A* and Pérez de Mendiola Etxezarraga X \\ Psychiatry Service, OSI Araba, Hospital Universitario Araba, Sede Santiago, Vitoria-Gasteiz, Araba, Basque Country, \\ Spain
}

\begin{abstract}
Background: The recurrent nature of Bipolar Disorder (BD) is the main cause of disability associated with the illness. Despite the proliferation of drugs approved for the maintenance phase of $\mathrm{BD}$, the relapse rate is still high. The combination of drugs, especially the potentiation of mood-stabilizers with second generation antipsychotics, may reduce the risk of relapse and rehospitalization. However, studies on the efficacy of specific combinations are scarce.
\end{abstract}

Case presentation: The clinical case of a 28 -year-old woman, who is involuntarily admitted to an Acute Psychiatric Unit, is presented. She suffers a manic postpartum episode with mixed and psychotic features. During the hospitalization, she is successfully treated with the combination of lithium plus olanzapine. In the discussion, a concise narrative review of the scientific literature on the efficacy of such a combination in BD is made.

Conclusions: The association of lithium plus olanzapine is one of the combinations with most evidence on its efficacy in $\mathrm{BD}$, especially in mixed-featured episodes. Tolerability concerns should not be an obstacle to its use, although they must be considered.

\section{Keywords}

Lithium plus olanzapine, Bipolar disorder, Combination therapies, Maintenance phase, Mixed features

\section{Introduction}

Bipolar Disorder (BD) is a chronic and recurrent illness that affects more than $1 \%$ of the global population $[1,2]$. It is one of the main causes of disability among young people, because it can lead to significant cognitive and functional impairment, as well as an increase in mortality, associated with cardiovascular disease and suicide $[3,4]$. The recurrent nature of bipolar disorder is the most determining factor in the long-term prognosis and therefore, implementing an effective maintenance treatment to prevent relapses is crucial [5]. Nonetheless, recurrence rates in BD remain high, with two-year relapse rates of $50 \%$ [6]. In women with $B D$, the postpartum is a particularly critical period. According to a recent meta-analysis, the overall postpartum relapse risk is $37 \%$ in women with $\mathrm{BD}$ but $66 \%$ in those who were medication-free during pregnancy [7].

In recent years, there has been a clear trend towards the use of combination treatments in $\mathrm{BD}$, especially those made up of second-generation antipsychotics with mood stabilizers [8-11]. This practice is supported by clinical experience and several recent studies $[6,11,12]$. Among all possible combinations, the sum of lithium and olanzapine could be particularly effective, both in the acute phase and in the maintenance phase of BD [13-16]. The following is a real clinical case, wherein this therapeutic combination is used to successfully treat a severe manic episode with psychotic and mixed features during the puerperium. Later, in the discussion, a narrative review of the literature on this topic is conducted.

\footnotetext{
*Corresponding author: Dr. Aitzol Miguélez Rodríguez, Psychiatry resident, OSI Araba, Hospital Universitario Araba, Sede Santiago, Psychiatry Service, Vitoria-Gasteiz, Araba, Basque Country, Spain; Tel: +34 945007764. Orcid ID: 00000002-8209-6629

Accepted: June 18, 2021

Published online: June 21, 2021

Citation: Rodríguez MA, Etxezarraga PMX, et al. (2021) Lithium plus Olanzapine as One of the Most Effective Combinations for Bipolar Disorder. A Case Report and a Concise Review of the Literature. J Psychiatry Treat Res 3(1):65-69
} 
Citation: Rodríguez MA, Etxezarraga PMX, et al. (2021) Lithium plus Olanzapine as One of the Most Effective Combinations for Bipolar Disorder. A Case Report and a Concise Review of the Literature. J Psychiatry Treat Res 3(1):65-69

\section{Case Description}

A 28-year-old woman is involuntarily admitted to an Acute Psychiatric Unit at a third-level hospital for behavioral abnormalities. She presents expansive and irritable mood, racing thoughts, inflated-self-esteem, hyperactivity, rampant purchases, and decreased need for sleep. The patient claims to have special abilities and that her family and friends want to harm her out of envy. She also exhibits prominent dysphoria, hopelessness, and thoughts of death. These symptoms are causing the patient serious social and work disruptions, due to aggressive and inappropriate behaviors.

Her medical history highlights a vacuum-assisted delivery two months before. Regarding psychiatric history, a previous admission due to a psychotic episode in the context of multiple drug abuse (alcohol, cannabis, cocaine, amphetamine...) is reported two years earlier. That episode was successfully treated with $300 \mathrm{mg}$ of aripiprazole long acting injectable (LAI) and the final diagnosis was a Substance-Induced Psychotic Disorder. During the subsequent follow-up, the patient suffered a depressive episode, which was treated with escitalopram. Few months later, when she became aware of the unplanned pregnancy and found herself clinically stable, she discontinued all psychopharmacological treatment.-

The current episode started in the postpartum and progressively had worsened within 2 months. At this time, in addition to mood-congruent and incongruent delusions, she presents affective symptoms of both poles, manic and depressive, with a predominance of the former. According to DSM-5 classification [17], this clinical picture corresponds to a manic episode with psychotic and mixed features. Regarding the complementary tests (urinalysis and complete blood analysis), the only pathological finding is the positive detection of cannabis in urine. The patient recognizes a sporadic and recreational use of cannabis (few times a month since adolescence) and she denies the use of other substances at present. A neuroimaging study is not performed because a cranial scan (computerized tomography) was made in the previous admission a year before, which was completely normal.

During current hospital admission, combination treatment with olanzapine plus lithium is started, until reaching a daily dose of $800 \mathrm{mg}$ of lithium ( $0.9 \mathrm{mEq} / \mathrm{L}$ serum level) and $20 \mathrm{mg}$ of olanzapine. Adequate tolerance and progressive remission of the symptoms are observed, obtaining a mood-stability, biorhythms restoration and functional recovery in a period of one month. $2 \mathrm{~kg}$ weight gain (with a BMI of $24.2 \mathrm{~kg} / \mathrm{m}^{2}$ ) was reported as a side effect. Young Mania Rating Scale shows a score of 40 at baseline and 7 at discharge from the hospital. The definitive diagnosis is Bipolar I disorder, current or most recent episode manic, with psychotic and mixed features, with peripartum onset (296.44 DSM-5, F31.2 ICD-10) [17].

Regarding the differential diagnosis, it is not considered a substance-induced disorder since the patient does not present the symptoms during or shortly after cannabis intoxication or withdrawal. Besides, the symptoms persisted during a significant period after cessation of drug use. The history of past depressive episode and the development of the symptoms during the postpartum are also helpful aspects for differential diagnosis.

\section{Discussion}

The clinical case described is a paradigmatic example of a postpartum onset manic episode with psychotic and mixed characteristics, successfully treated with lithium plus olanzapine. In order to study the effectiveness of this combination in $\mathrm{BD}$, a comprehensive literature search was conducted using the MEDLINE database in January 2021. Search terms included the following: (Bipolar disorder) and (lithium) and (olanzapine). The main findings of the bibliographic retrieval are summarized below.

Antipsychotics, particularly olanzapine, have shown to be more effective, essentially faster, than mood stabilizers in the manic episodes $[18,19]$. Olanzapine also turns out to be one of the drugs with greater acceptability and with the lowest discontinuation rate in the manic episodes $[18,20]$. Furthermore, olanzapine monotherapy has proven to be effective in preventing recurrences, surpassing the rest of antipsychotics in this regard [21]. Its prophylactic action is eminently antimanic, although with a polarity index more balanced than other antipsychotics, for example aripiprazole or risperidone [4,22], which implies a greater effect of olanzapine in the prevention of depressive relapses.

The efficacy of olanzapine in episodes with mixed features deserves special mention. In fact, in the latest clinical guidelines, it is listed as the treatment of choice for the mixed states [23-25]. This aspect is highly relevant, since mixed symptoms are the most common in the $\mathrm{BD}$, they are the most difficult to treat and are associated with a worse prognosis, higher suicide rate and substance abuse [24,26-28].

On the other hand, lithium is still considered the Gold Standard treatment for the BD $[29,30]$. It prevents manic, depressive, and mixed episodes effectively, with a robust evidence [26,31], supported by recent both controlled and observational studies [32,33]. Independently of its mood-stabilizing effect, lithium has unique anti-suicide and neuroprotective properties [34]. In addition, it is the only drug that has been shown to be highly effective for the acute and maintenance treatment of postpartum psychosis [35].

Therefore, if we consider the favorable data for the use of lithium and olanzapine in monotherapy in the BD, it would be expected that the combination of both could provide additional benefits. Four recent papers support this hypothesis. In the first of these [13], the addition of olanzapine to lithium or valproate significantly increased the efficacy of the treatment in manic or mixed episodes. In the second study [14], symptomatic remission was found to be longer with combination therapy (olanzapine plus lithium or valproate) than with monotherapy (lithium or valproate alone), in a group of patients who had recently achieved remission after a manic or mixed episode. Similar results were found in the third controlled trial [15], which showed that in patients who maintained combination therapy (olanzapine plus lithium or valproate) the time to relapse was longer, compared to those who discontinued antipsychotic treatment. The last 
Citation: Rodríguez MA, Etxezarraga PMX, et al. (2021) Lithium plus Olanzapine as One of the Most Effective Combinations for Bipolar Disorder. A Case Report and a Concise Review of the Literature. J Psychiatry Treat Res 3(1):65-69

one is a cohort study [16], in which it is suggested that the rehospitalization rate, after a manic episode, is significantly lower in patients with combination therapy (olanzapine plus lithium or valproate), compared to lithium monotherapy.

The main limitations for the use of the combined treatment are the problems of tolerability and the increased risk of adverse effects [11,36]. However, this does not always lead to discontinuation of the treatment, since the benefit perceived by the patient, in terms of symptomatic or syndromic remission, could prevail over the negative effects of the treatment [6]. This would explain the aforementioned increase in the use of combination therapies in real-world practice. Furthermore, there are effective strategies to prevent some of the most worrisome adverse effects, such as weight gain and diabetes in the case of Olanzapine [37] or renal dysfunction in the case of lithium $[38,39]$. It should not be forgotten that the alternatives are not risk free: quetiapine has a similar metabolic and sedative profile to Olanzapine [40], risperidone causes more extrapyramidal and sexual side-effects [41] and valproate is related to a high teratogenic risk [42]. Despite its side effects, olanzapine presents a lower risk of discontinuation than aripiprazole [43] and is one of the best rated antipsychotics, both by psychiatrists and by the patients [44]. Concerning lithium use, it is now recognized that its adverse effects, specifically the most serious (renal and teratogenic), could have been overestimated in the oldest studies [38,45], which could have generated an excessively negative perception of its "toxicity" $[34,46,47]$.

The study's main limitation is the narrative nature of the review, with the risk of selection bias that this implies. However, given the sparse studies evaluating the effectiveness of the association of lithium and olanzapine in $\mathrm{BD}$, we consider that it is an adequate approach to the topic. This review raises the need for future research comparing the efficacy of different combination treatments, both with each other and with monotherapy.

\section{Conclusions}

In summary, as reflected in the current clinical guidelines and real-world clinical practice, the use of combination therapies (second-generation antipsychotics associated with mood stabilizers) is a suitable treatment in BD [2325]. It could be more effective than monotherapy in certain clinical situations, as severe episodes with psychotic or mixed features. Considering the results of this review, the association of olanzapine and lithium could be one of the combinations with the most scientific evidence in its favor. Therefore, we conclude that it is an appropriate therapeutic strategy in resistant or difficult cases, like the one exposed, despite the reasonable tolerability concerns.

\section{Authors' Disclosures and Acknowledgments}

The authors of the present article declare the absence of financial support for the conduction of the present research.

Both authors declare that the elaboration, review and submission process of the present manuscript has been equitably carried out. Both authors have contributed equitably in conceptualization, investigation, project administration, supervision, visualization, writing the original draft, reviewing and editing of this article.

Both authors clearly state the absence of conflicts of interest that could inappropriately impact or influence the research and interpretation of the findings.

There are not additional acknowledgments to be declared related to the elaboration of the present report.

All authors have seen and approved the final version of the manuscript being submitted. They warrant that the article is the authors' original work, hasn't received prior publication and isn't under consideration for publication elsewhere apart from having strictly complied APA ethical standards.

\section{References}

1. Pini S, de Queiroz V, Pagnin D, et al. (2005) Prevalence and burden of bipolar disorders in European countries. Eur Neuropsychopharmacol 15: 425-434.

2. Carvalho AF, Firth J, Vieta E (2020) Bipolar disorder. N Engl J Med 383: 58-66.

3. Grande I, Berk M, Birmaher B, et al. (2016) Bipolar disorder. Lancet 387: 1561-1572.

4. Vieta E, Berk M, Schulze TG, et al. (2018) Bipolar disorders. Nat Rev DisPrimers 4: 18008.

5. González-Pinto A, Barbeito S, Alonso M, et al. (2011) Poor longterm prognosis in mixed bipolar patients: $10-$ Year outcomes in the Vitoria prospective naturalistic study in Spain. J Clin Psychiatry 72: 671-676.

6. Wingard L, Brandt L, Bodén R, et al. (2019) Monotherapy vs. combination therapy for post mania maintenance treatment: $A$ population based cohort study. Eur Neuropsychopharmacol 29: 691-700.

7. Wesseloo R, Kamperman AM, Munk-Olsen T, et al. (2016) Risk of postpartum relapse in bipolar disorder and postpartum psychosis: A systematic review and meta-analysis. Am J Psychiatry 173: 117-127.

8. Bjorklund L, Horsdal HT, Mors O, et al. (2016) Trends in the psychopharmacological treatment of bipolar disorder: A nationwide register-based study. Acta Neuropsychiatr 28: 7584.

9. Chang CM, Wu CS, Huang YW, et al. (2016) Utilization of psychopharmacological treatment among patients with newly diagnosed bipolar disorder from 2001 to 2010. J Clin Psychopharmacol 36: 32-44.

10. Kessing LV, Vradi E, Andersen PK (2016) Nationwide and population-based prescription patterns in bipolar disorder. Bipolar Disord 18: 174-182.

11. Buoli M, Serati M, Altamura AC (2014) Is the combination of a mood stabilizer plus an antipsychotic more effective than mono-therapies in long-term treatment of bipolar disorder? A systematic review. J Affect Disord 152-154: 12-18.

12. Hochman E, Krivoy A, Schaffer A, et al. (2016) Antipsychotic adjunctive therapy to mood stabilizers and 1-year rehospitalization rates in bipolar disorder: A cohort study. Bipolar Disord 18: 684-691. 
Citation: Rodríguez MA, Etxezarraga PMX, et al. (2021) Lithium plus Olanzapine as One of the Most Effective Combinations for Bipolar Disorder. A Case Report and a Concise Review of the Literature. J Psychiatry Treat Res 3(1):65-69

13. Tohen M, Chengappa KN, Suppes T, et al. (2002) Efficacy of olanzapine in combination with valproate or lithium in the treatment of mania in patients partially nonresponsive to valproate or lithium monotherapy. Arch Gen Psychiatry 59: 6269.

14. Tohen M, Chengappa KN, Suppes T, et al. (2004) Relapse prevention in bipolar I disorder: 18-month comparison of olanzapine plus mood stabiliser v. mood stabiliser alone. $\mathrm{Br} \mathrm{J}$ Psychiatry184: 337-345.

15. Yatham L, Beaulieu S, Schaffer A, et al. (2016) Optimal duration of risperidone or olanzapine adjunctive therapy to mood stabilizer following remission of a manic episode: A CANMAT randomized double-blind trial. Mol Psychiatry 21: 1050-1056.

16. Wingard L, Boden R, Brandt L, et al. (2017) Reducing the rehospitalization risk after a manic episode: A population based cohort study of lithium, valproate, olanzapine, quetiapine and aripiprazole in monotherapy and combinations. J Affect Disord 217: $16-23$

17. American Psychiatric Association (2013) Diagnostic and statistical manual of mental disorders. ( $5^{\text {th }}$ edn), American Psychiatric Association, Washington, DC.

18. Cipriani A, Barbui C, Salanti G, et al. (2011) Comparative efficacy and acceptability of antimanic drugs in acute mania: A multipletreatments meta-analysis. Lancet 378: 1306-1315.

19. Yildiz A, Vieta E, Leucht S, et al. (2011) Efficacy of antimanic treatments: Meta-analysis of randomized, controlled trials. Neuropsychopharmacology 36: 375-89.

20. Yildiz A, Nikodem M, Vieta E, et al. (2015) A network metaanalysis on comparative efficacy and all-cause discontinuation of antimanic treatments in acute bipolar mania. Psychol Med. 45: 299-317.

21. Baldessarini RJ, Tondo L, Vázquez GH (2019) Pharmacological treatment of adult bipolar disorder. Mol Psychiatry 24: 198-217.

22. Popovic D, Reinares M, Goikolea JM, et al. (2012) Polarity index of pharmacological agents used for maintenance treatment of bipolar disorder. Eur Neuropsychopharmacol 22: 339-346.

23. Fountoulakis KN, Grunze H, Vieta E, et al. (2016) The International College of Neuro-Psychopharmacology (CINP) treatment guidelines for Bipolar disorder in adults, part 3: The clinical guidelines. Int J Neuropsychopharmacol 20: 180-195.

24. Grunze H, Vieta E, Goodwin GM, et al. (2018) The World Federation of Societies of Biological Psychiatry (WFSBP) Guidelines for the Biological Treatment of Bipolar Disorders: Acute and long-term treatment of mixed states in bipolar disorder. World J Biol Psychiatry 19: 2-58.

25. Yatham LN, Kennedy SH, Parikh SV, et al. (2018) Canadian Network for Mood and Anxiety Treatments (CANMAT) and International Society for Bipolar Disorders (ISBD) 2018 guidelines for the management of patients with bipolar disorder. Bipolar Disord 20: 97-170.

26. González-Pinto A, López-Peña $P$, Bermúdez-Ampudia $C$, et al (2018) Can lithium salts prevent depressive episodes in the real world? Eur Neuropsychopharmacol 28: 1351-1359.

27. Malhi GS, Fritz K, Elangovan P, et al. (2019) Mixed states: Modelling and management. CNS Drugs 33: 301-313.

28. Vieta E, Valentí M (2013) Mixed states in DSM-5: Implications for clinical care, education, and research. J Affect Disord 148: 28-36.
29. Kessing LV, Bauer M, Nolen WA, et al. (2018) Effectiveness of maintenance therapy of lithium vs. other mood stabilizers in monotherapy and in combinations: A systematic review of evidence from observational studies. Bipolar Disord 20: 419431.

30. Severus E, Bauer M, Geddes J (2018) Efficacy and effectiveness of lithium in the long-term treatment of bipolar disorders: An update 2018. Pharmacopsychiatry 51: 173-176.

31. Miura T, Noma H, Furukawa TA, et al. (2014) Comparative efficacy and tolerability of pharmacological treatments in the maintenance treatment of bipolar disorder: A systematic review and network meta-analysis. Lancet Psychiatry 1: 35135-35139.

32. Berk M, Daglas R, Dandash O, et al. (2017) Quetiapine v. lithium in the maintenance phase following a first episode of mania: Randomised controlled trial. Br J Psychiatry 210: 413-421.

33. Lähteenvuo M, Tanskanen A, Taipale $\mathrm{H}$, et al. (2018) Real world effectiveness of pharmacologic treatments for the prevention of rehospitalization in a finnish nationwide cohort of patients with bipolar disorder. JAMA Psychiatry 75: 347-355.

34. Rybakowski JK (2018) Challenging the negative perception of lithium and optimizing its long-term administration. Front Mol Neurosci. 11: 349.

35. Bergink V, Burgerhout KM, Koorengevel KM, et al. (2015) Treatment of psychosis and mania in the postpartum period. Am J Psychiatry 172: 115-123.

36. Ogawa Y, Tajika A, Takeshima N, et al. (2014) Mood stabilizers and antipsychotics for acute mania: A systematic review and meta-analysis of combination/augmentation therapy versus monotherapy. CNS Drugs 28: 989-1003.

37. Siskind D, Hahn M, Correll CU, et al. (2019) Glucagon-like peptide-1 receptor agonists for antipsychotic-associated cardiometabolic risk factors: A systematic review and individual participant data meta-analysis. Diabetes Obes Metab 21: 293302.

38. Nielsen RE, Kessing LV, Nolen WA, et al. (2018) Lithium and renal impairment: A review on a still hot topic. Pharmacopsychiatry 51: 200-205.

39. Schoot TS, Molmans THJ, Grootens KP, et al. (2020) Systematic review and practical guideline for the prevention and management of the renal side effects of lithium therapy. Eur Neuropsychopharmacol 31: 16-32.

40. Hayes JF, Marston L, Walters K, et al. (2016) Lithium vs. valproate vs. Olanzapine vs. quetiapine as maintenance monotherapy for bipolar disorder: A population-based UK cohort study using electronic health records. World Psychiatry 15: 53-58.

41. Leucht S, Cipriani A, Spineli L, et al. (2013) Comparative efficacy and tolerability of 15 antipsychotic drugs in schizophrenia: A multiple-treatments meta-analysis. Lancet 382: 951-962.

42. (2018) Newmeasures to avoid valproate exposure in pregnancy endorsed. Member State representatives agree new restrictions and pregnancy prevention programme. European Medicines Agency.

43. Huhn M, Nikolakopoulou A, Schneider-Thoma J, et al. (2019) Comparative efficacy and tolerability of 32 oral antipsychotics for the acutetreatment of adults with multi-episode schizophrenia: A systematic review andnetwork meta-analysis. Lancet 394: 939-951. 
Citation: Rodríguez MA, Etxezarraga PMX, et al. (2021) Lithium plus Olanzapine as One of the Most Effective Combinations for Bipolar Disorder. A Case Report and a Concise Review of the Literature. J Psychiatry Treat Res 3(1):65-69

44. Campos P, Carrió M, Vicedo A, et al. (2020) Actitud de los psiquiatras respecto al uso de olanzapina en la práctica clínica: Una encuesta nacional. Psiquiatría Biológica 27: 3-8.

45. Fornaro M, Maritan E, Ferranti R, et al. (2020) Lithium exposure during pregnancy and the postpartum period: A systematic review and meta-analysis of safety and efficacy outcomes. Am J Psychiatry 177: 76-92.
46. Zivanovic O (2017) Lithium: A classic drug-Frequently discussed, but, sadly, seldom prescribed. Aust N Z J Psychiatry 51: 886-896.

47. Pérez de Mendiola X, Hidalgo-Mazzei D, Vieta E, et al. (2021) Overview of lithium's use: A nationwide survey. Int J Bipolar Disord 9: 10 